\title{
Review of: "Ultrasound-assisted Maillard reaction of ovalbumin/xylose: The enhancement of functional properties and its mechanism"
}

\author{
Yuqing Duan ${ }^{1}$ \\ 1 Jiangsu University
}

Potential competing interests: The author(s) declared that no potential competing interests exist.

This paper is well designed, it is very enlightening to my research. I'm very interested in it. 